\title{
EXISTENCE OF SOLUTIONS TO NONSELFADJOINT BOUNDARY VALUE PROBLEMS FOR ORDINARY DIFFERENTIAL EQUATIONS
}

\author{
L. Cesari \\ Department of Mathematics, University of Michigan, Ann Arbor, Michigan 48109, U.S.A. \\ and \\ T. T. BOWMAN \\ Department of Mathematics, University of Florida, Gainesville, Florida 32611, U.S.A.
}

(Received 1 April 1984; received for publication 25 February 1985)

\begin{abstract}
Key words and phrases: Existence of solutions, nonlinear ordinary differential equations, nonselfadjoint boundary value problems, the alternative method, uniform topology, finite dimensional kernels and cokernels.
\end{abstract}

\section{INTRODUCTION}

IN THE present paper we prove existence theorems for nonselfadjoint real boundary value problems for semilinear ordinary differential equations

$$
\begin{aligned}
& x^{(n)}+p_{1}(t) x^{(n-1)}+\ldots+p_{n}(t) x=f(t)+g(t, x(t)), 0<t<a, \\
& \sum_{j=0}^{n-1} a_{h j} x^{(j)}(0)+b_{h j} x^{(j)}(a)=0, h=1, \ldots, m,
\end{aligned}
$$

where the coefficients $p_{s}(t)$ are continuous functions on $[0, a], p_{0}(t)>0$, and the $a_{h j}, b_{h j}$ are constants.

Our results show that, even for nonselfadjoint boundary value problems, a limited quantitative form of the Landesman and Lazer sufficient condition for existence holds. Moreover, further extensions are obtained of the recent form proposed by Shaw of the Landesman and Lazer theorem.

The point of departure of the present paper is the theorem of Landesman and Lazer [11], which represents a necessary and sufficient condition in order that a selfadjoint real elliptic partial differential equation of order $2, E x=f(t)+g(x), t \in G$, with $x=0$ on $\partial G$, has a weak solution $x \in W_{0}^{1.2}(G)$, where $G$ is a bounded domain in $\mathbb{R}^{n}, g: \mathbb{R} \rightarrow \mathbb{R}$ is continuous with finite limits $g(-\infty) \neq g(+\infty)$, and $f \in L_{2}(G)$.

This remarkable theorem was extended by Williams [15] and by De Figueiredo [10] to elliptic problems of order $2 n$ and nonlinearity $g$ depending on derivatives of orders $<2 n-1$. The same theorem was then extended by Shaw [14] to nonselfadjoint boundary value problems for partial differential equations with $p=q<\infty, p=\operatorname{dim} \operatorname{ker} E, q=\operatorname{dim} \operatorname{ker} E^{*}$, where $E^{*}$ is the adjoint of $E$, provided corresponding elements of ker $E$ and $\operatorname{ker} E^{*}$, share the same regions of positivity and negativity. In particular, for ordinary differential equations with $n=1, p=q=1$, this condition is always satisfied, and the Landesman and Lazer theorem holds. For their results, Landesman and Lazer, as well as Williams and Shaw made use of ideas from the alternative method (see, e.g. [6]). 
In the present paper, we first analyze further the alternative method, for the case where $\propto>p \geqslant q \geqslant 0$, and there is an orthonormal basis $\left(\omega_{1}, \ldots, \omega_{q}\right)$ of ker $E^{*}$ and orthornormal elements $\phi_{1}, \ldots, \phi_{q}$ in ker $E$ such that the $q \times q$ matrix $M=\left(\omega_{s}, \phi_{i}\right), s, i=1, \ldots, q$, is not singular. Thus, if in particular $\phi_{i}=\omega_{i}, i=1, \ldots, q$, then $M$ is the identity matrix and this condition is satisfied. Also, for $p=q=1$, if $\omega_{1}$ and $\phi_{1}$ share the same regions of positivity and negativity (Shaw's condition), then $M$ is a positive scalar.

Now the decompositions often used in the alternative method with $X=Y=L_{2}[0, a]$, say, $X=\operatorname{ker} E+X_{1}, Y=\operatorname{ker} E^{*}+Y_{1}$ are replaced by a further decomposition $X=X_{01}+$ $X_{02}+X_{1}, Y=Y_{0}+Y_{1}, Y_{0}=\operatorname{ker}^{*} E=\operatorname{sp}\left\langle\omega_{1}, \cdots, \omega_{q}\right\rangle, X_{01}=\operatorname{sp}\left\langle\phi_{1}, \cdots, \phi_{q}\right\rangle$, ker $E=$ $X_{01}+X_{02}, X_{1}=(\operatorname{ker} E)^{\perp}, Y_{1}=\left(\operatorname{ker} E^{*}\right)^{\perp}$, with usual projection operator $Q: Y \rightarrow Y, Q Y=$ $Y_{0}=\operatorname{ker} E^{*}, P: X \rightarrow X, P X=X_{0}=X_{01}+X_{02}$.

Under these assumptions, the map $S: \operatorname{ker} E^{*} \rightarrow \operatorname{ker} E$ with $S^{-1}(0)=\{0\}$, which is variously defined in the alternative method, can be so chosen that the map $S: \operatorname{ker} E^{*} \rightarrow X_{01}$ is linear, $1-1$, and onto, and $S Q: Y \rightarrow X_{01}$, restricted to $X_{01}$, is the identity.

On the basis of this particular map $S$, we present a new argument in dealing with the bifurcation equation $Q N x=0$. We then obtain a new existence theorem for problem (1), (2), with $\|f\|_{\infty}<c$, a suitable constant, which allows a remarkable freedom for the continuous Lipschitzian function $g$.

\section{THE LINEAR OPERATOR $E$ IN $L_{2}[0, a]$}

Here $J$ stands for a closed interval $[0, a]$ of the real line, and $D(T), R(T)$, ker $T$ denote the domain, the range, and the null space or kernel of a linear operator $T$, respectively. Also $\operatorname{sp}\left(\omega_{1}, \cdots, \omega_{m}\right)$ stands for the linear space spanned by $\omega_{1}, \cdots, \omega_{m}$, and $T \mid D_{0}$ denotes the restriction of the operator $T$ on a given set $D_{0} \subset D(T)$.

We consider the boundary value problem

$$
\begin{gathered}
\tau x \equiv x^{(n)}+p_{1}(t) x^{(n-1)}+\cdots+p_{n}(t) x=f(t)+g(t, x(t)), \quad t \in(0, a), \\
B_{i} x \equiv \sum_{j=0}^{n-1} a_{i j} x^{(j)}(0)+b_{i j} x^{(j)}(a)=0, \quad i=1, \cdots, m,
\end{gathered}
$$

where each coefficient $p_{i}(t)$ is of class $C^{n-i}$ in $[0, a], f \in L_{2}[0, a]$, and $g:[0, a] \times \mathbb{R} \rightarrow \mathbb{R}$ is a continuous function of $x$ for every $t$, and measurable in $t$ for every $x$. We shall also assume that $g(t, x)$ is bounded in $[0, a] \times \mathbb{R}$. In (2) we assume that the coefficients $a_{i j}, b_{i j}$ are real constants, and that the $m$ forms $B_{i}$ are linearly independent.

Let $S=L_{2}[0, a]$ with usual inner product $($,$) and norm \|\|_{2}$. Let $S^{(n)}$ denote the space of all functions $x(t), 0 \leqslant t \leqslant a$, which are $A C$ in $[0, a]$ together with $x^{\prime}, \cdots, x^{(n-1)}$, and $x^{(n)} \in$ $L_{2}[0, a]$, and we take in $S^{(n)}$ the norm

$$
\|x\|_{2}^{(n)}=\|x\|_{x}+\left\|x^{\prime}\right\|_{x}+\cdots+\left\|x^{(n-1)}\right\|_{x}+a^{-1}\left(\int_{0}^{a}\left(x^{(n)}(t)\right)^{2} \mathrm{~d} t\right)^{1 / 2}, \quad x \in S^{(n)} .
$$

Let $E$ denote the operator defined by $E x=\tau x$ with domain $D(E)=\left[x \in S^{(n)}, B_{i} x=0, i=\right.$ $1, \ldots, m]$ so that $E: D(E) \subset S \rightarrow S$.

The following statements are well known:

$D(E)$ is dense in $S$,

$E$ is a closed linear operator,

$R(E)$ is a closed linear subspace of $S$. 
We assume $x>p \geqslant q \geqslant 0$ where $p=\operatorname{dim} \operatorname{ker} E$ and $q=\operatorname{dim} \operatorname{coker} E=\operatorname{dim} \operatorname{ker} E^{*}$. Let $\phi_{1}, \cdots, \phi_{p}$ be an orthonormal basis for ker $E$. Now the restriction of $E$ to the space $D(E) \cap$ $(\text { ker } E)^{\perp}$ is a $1-1$ closed linear operator whose closed range is still $R(E)$. Hence, by the closed graph theorem, the inverse map $H=\left[E \mid D(E) \cap(\operatorname{ker} E)^{-}\right]^{-1}$ is a $1-1$ continuous linear operator with domain $R(E)$ and range $D(E) \cap($ ker $E$ ). Also,

$$
\begin{aligned}
& E H y=y \quad \text { for all } \quad y \in R(E), \\
& H E x=x-\sum_{i=1}^{p}\left(x, \phi_{i}\right) \phi_{i} \quad \text { for all } \quad x \in D(E),
\end{aligned}
$$

where $(u, v)$ denote the usual inner product in $L_{2}[0, a]$ and then $\|u\|_{2}=(u, u)^{1 / 2}$. By Fredholm alternative theorem we also know that $y \in R(E)$ if and only if $y \perp \operatorname{ker} E^{*}$, or ker $E^{*}=R(E)^{\perp}$.

We have just stated that the linear operator $H: R(E) \rightarrow D(E) \cap(\text { ker } E)^{-}$is continuous, hence bounded, in the norm $L_{2}$ in $R(E)$ and the norm $\|x\|_{2}^{(n)}$ in $D(E) \cap(\operatorname{ker} E)^{-}$, and the statement holds that for any $f \perp$ ker $E^{*}$ the only solution $H f$ of (1), (2) which is orthogonal to ker $E$ satisfies

$$
\|H f\|^{(n)} \leqslant \gamma\|f\|_{2}
$$

where $\gamma$ is a suitable constant independent of $f$. We note that, since $\|x\|_{2}^{(n)}$ generates a stronger topology on $D(E) \cap(\text { ker } E)^{\perp}$ than the norm $\|x\|_{2}$, then $H$ can be viewed also as a continuous map of $R(E)$ to $S$ with both having the $L_{2}$ norm.

We denote by $N$ the Nemitsky operator defined by $N x=f(t)+g(t, x(t))$ for $x \in S, f \in L_{2}$, so that $g(t, x(t))$ is measurable and bounded, and $N: S \rightarrow S, D(N)=S \supset S^{(n)}=D(E)$. Problem (1), (2) now takes the form

$$
E x=N x, x \in D(E) .
$$

Let $P: S \rightarrow S$ and $Q: S \rightarrow S$ denote the orthogonal projections of $S$ onto ker $E$ and ker $E^{*}$ respectively, namely

so that

$$
\begin{aligned}
& (P x)(t)=\sum_{i=1}^{p}\left(x, \phi_{i}\right) \phi_{i}(t) \quad \text { for } \quad x \in S, \\
& (Q y)(t)=\sum_{s=1}^{q}\left(y, \omega_{s}\right) \omega_{s}(t) \quad \text { for } \quad y \in S,
\end{aligned}
$$

$$
\begin{aligned}
& P P=P, Q Q=Q, P S=\operatorname{ker} E, Q S=\operatorname{ker} E^{*}=\operatorname{coker} E, \\
& (I-P) S=(\operatorname{ker} E)^{\perp},(I-Q) S=\left(\operatorname{ker} E^{*}\right)^{\perp}=R(E) .
\end{aligned}
$$

It is well known that equation $E x=N x$ splits into the system of auxiliary and bifurcation equations

$$
x=P x+H(I-Q) N x, Q N x=0, x \in S .
$$

\section{A PARTICULARIZATION IN $C[0, a]$}

We consider now a particularization of (1), (2), namely we assume that $f:[0, a] \rightarrow \mathbb{R}$ and $g:[0, a] \times \mathbb{R} \rightarrow \mathbb{R}$ are continuous functions, and that $g$ is bounded in $[0, a] \times \mathbb{R}$. We further assume that the coefficients $p_{1}, \cdots, p_{n}$ in (1) are continuous functions in $[0, a]$. 
Let $X$ and $Y$ be copies of $C=C[0, a]$. Then the Nemitsky operator $N x=f(t)+g(t, x(t))$ is defined on all of $X$ with values in $Y$, or $N: X \rightarrow Y$. We may restrict $D(E)$ to the new set

$$
D_{0}(E)=\left[x \in S^{(n)}, x^{(n)} \text { continuous, } B_{i} x=0, i=1, \cdots, m\right] \text {, }
$$

so that $E: D_{0}(E) \subset X \rightarrow Y$,

Let us prove that

$$
E: D_{0}(E) \cap(\operatorname{ker} E)^{\perp} \rightarrow R(E) \cap C, \quad H: R(E) \cap C \rightarrow D_{0}(E) \cap(\operatorname{ker} E)^{\perp}
$$

and that $R(E) \cap C$ is closed in $C$. Indeed $R(E)$ is closed in $L_{2}$, convergence in $C$ implies convergence in $L_{2}$, and hence $R(E) \cap C$ is closed in $C$. Also, $E: D(E) \rightarrow R(E)=S \cap$ (ker $\left.E^{*}\right)^{\perp}$, and by the definition of $E, E x$ is continuous in $[0, a]$ since $x, x^{\prime}, \cdots, x^{(n)}, p_{1}, \cdots, p_{n}$ are all continuous, and thus $E: D_{0}(E) \rightarrow S \cap C \cap\left(\operatorname{ker} E^{*}\right)^{\perp}=R(E) \cap C$. Finally, for $y \in$ $R(E) \cap C$, then $x=H y \in D(E) \cap(\operatorname{ker} E)^{\perp}$, certainly $x, x^{\prime} \cdots, x^{(n-1)}$ are $A C$, and $x^{(n)}=y-$ $p_{n} x-\cdots-p_{1} x^{(n-1)}$, where $p_{1}, \cdots, p_{n}$ are continuous; hence $x^{(n)}$ is continuous and $x=$ $H y \in D_{0}(E)$. Thereby, (6) is proved.

As usual we take now $X_{0}=P X, X_{1}=(I-P) X, Y_{0}=Q Y, Y_{1}=(I-Q) Y$, where $X=$ $Y=S$. Since $\phi_{1}, \cdots, \phi_{p}, \omega_{1}, \cdots, \omega_{q}$ are continuous functions, we have decompositions $C=X=X_{0}+X_{1}, C=Y=Y_{0}+Y_{1}$, and $Y_{1}=R(E) \cap C, X_{1}=C \cap(\operatorname{ker} E)^{\perp}, X_{0}=\mathrm{kcr}$ $E, Y_{0}=\operatorname{ker} E^{*}$, and equation $E x=N x, x \in D_{0}(E)$, still splits into the two equations

$$
x=P x+H(I-Q) N x, \quad Q N x=0, \quad x \in X .
$$

Any solution $x \in X$ of this system is an element $x=x_{0}+x_{1}, x_{0}=P_{x} \in \operatorname{ker} E, x_{1} \in D_{0}(E)$ $\cap(\operatorname{ker} E)^{\perp}$ and is a solution of problem (1), (2). The uniform topology in $C=X=Y$ is defined by the usual Sup norm in $[0, a]$, or \|\|$_{\infty}$, and, in this norm, we cannot expect $P$ and $Q$ to have norm one, but necessarily $\geqslant 1$ :

$$
\|P\|=c_{1}, \quad\|I-P\|=c_{2}, \quad\|Q\|=c_{3}, \quad\|I-Q\|=c_{4}, \quad\|H\|=L .
$$

Actually, we shall denote by these letters constants such that $\|P x\|_{x} \leqslant c_{1}\|x\|_{x},\|(I-P) x\|_{x} \leqslant$ $c_{2}\|x\|_{x}$ for all $x \in X,\|Q y\|_{x} \leqslant c_{3}\|y\|_{x},\|(I-Q) y\|_{\infty} \leqslant c_{4}\|y\|_{x}$ for all $y \in Y$, and $\|H y\|_{x} \leqslant L\|y\|_{x}$ for all $y \in Y_{1}$.

Now

$$
X_{0}=\operatorname{ker} E=\operatorname{sp}\left(\phi_{1}, \cdots, \phi_{p}\right), Y_{0}=\operatorname{ker} E^{*}=\operatorname{sp}\left(\omega_{1}, \cdots, \omega_{q}\right), p \geqslant q,
$$

and we split $X_{0}$ into a direct sum of a space $X_{01}$ of dimension $q$ and its complement in $X_{0}$. Actually, by possibly changing bases and their indexing, we can always assume that

$$
X_{01}=\operatorname{sp}\left(\phi_{1}, \cdots, \phi_{q}\right), X_{02}=\operatorname{sp}\left(\phi_{q+1}, \cdots, \phi_{p}\right), X_{0}=X_{01}+X_{02} .
$$

Let $M$ denote the $q \times q$ matrix $M=\left[\left(\omega_{s}, \phi_{i}\right), s, i=1, \cdots, q\right]$, and we assume that $M$ is nonsingular. For instance, if $\omega_{i}=\phi_{i}, i=1, \cdots, q$, then $M$ is the identity matrix.

We shall use the notational convention to denote briefly by $\sigma$ any given vector $\sigma=$ $\operatorname{col}\left(\sigma_{1}, \cdots, \sigma_{q}\right)$. We now define a linear map $S: Y_{0} \rightarrow X_{01}$. Indeed, for every $y \in Y_{0}$ we have $y=\sum_{s=1}^{q} d_{s}^{*} \omega_{s}$ with $d_{s}^{*}=\left(y, \omega_{s}\right)$, since the $\omega_{s}$ are orthogonal, and we take $S y=\sum_{i=1}^{q} d_{i} \phi_{i}$ with $d=M^{-1} d^{*}$. 
(3.i) $S^{-1}(0)=\{0\}$. Indeed, if $y \in S^{-1}(0)$, then $S y=0$, that is, $d=0$, hence $d^{\#}=0$ since $M$ is nonsingular, and $y=0$.

We note now that $X_{01} \subset X=Y$; hence, $Q: Y \rightarrow Y$ is defined on $X_{01}$.

(3.ii) $S Q x=x$ for all $x \in X_{01}$. In other words, $S Q=I$ is the identity on $X_{01}$.

Indeed, if $x \in X_{01}$ then $x=\sum_{i=1}^{q} c_{i} \phi_{i}, c=\left(x, \phi_{i}\right)$, and

$$
Q x=\sum_{s=1}^{q}\left(x, \omega_{s}\right) \omega_{s}=\sum_{s=1}^{q}\left(\sum_{i=1}^{q} c_{i} \phi_{i}, \omega_{s}\right) \omega_{s}=\sum_{i=1}^{q}\left(\sum_{i=1}^{q}\left(\omega_{s}, \phi_{i}\right) c_{i}\right) \omega_{s}=\sum_{s=1}^{q} c_{s}^{*} \omega_{s}
$$

with $c^{*}=M c$, and then $S Q x=\sum_{i=1}^{q} c_{i}^{\prime} \phi_{i}$ with $c^{\prime}=M^{-1}(M c)=c$.

Now $S Q$, considered as a linear operator $S Q: Y \rightarrow X_{0}$ has a norm (in the uniform topologies of $Y$ and $X$ ) we shall denote by $c_{5}$. Actually, we shall denote by $c_{5}$ a constant such that $\|S Q y\|_{x}$ $\leqslant c_{5}\|y\|_{x}$ for all $y \in Y$.

\section{THE EXISTENCE THEOREM}

Let us consider the set

$$
\Omega=S_{0} \times S_{1}, S_{0}=\left[x_{01} \in X_{01} \mid\left\|x_{01}\right\|_{\infty} \leqslant R_{0}\right], S_{1}=\left[x_{1} \in X_{1} \mid\left\|x_{1}\right\|_{x} \leqslant r\right]
$$

for given numbers $R_{0}, r>0$. Let $x_{02} \in X_{02}$ be arbitrary, take $x=x_{01}+x_{02}+x_{1}, x_{01} \in S_{0}$, $x_{1} \in S_{1}$, and let us consider the transformation $T: x_{01}+x_{02}+x_{1} \rightarrow \bar{x}_{01}+\bar{x}_{02}+\bar{x}_{1}$ defined by

$$
T:\left\{\begin{array}{l}
\bar{x}_{1}=H(I-Q)\left[f(t)+g\left(t, x_{01}+x_{02}+x_{1}\right)\right] \\
\bar{x}_{01}=x_{01}-k S Q\left[f(t)+g\left(t, x_{01}+x_{02}+x_{1}\right)\right], \quad \bar{x}_{02}=x_{02} .
\end{array}\right.
$$

Since we shall treat $x_{02}$ as an arbitrary element of $X_{02}$, it is convenient to consider $T$ as a transformation $\bar{x} \rightarrow \overline{\bar{x}}$ with $\bar{x}=\left(x^{*}, x_{1}\right), \widetilde{\bar{x}}=\left(\bar{x}^{*}, \bar{x}_{1}^{*}\right)$. With this convention, it is immediate that $T$ maps $\Omega$ into $X_{01}+X_{1}$.

We assume now that for some constants $R_{0}, r, k, c, C, D, \rho>0$ and $r^{\prime} \geq 0$, we have

$$
\begin{gathered}
|f(t)| \leqslant c \text { for } t \in J=[0, a], \\
|g(t, z)| \leqslant C \text { for }(t, z) \in J \times \mathbb{R},|z| \leqslant R_{0}+r+r^{\prime}, \\
|g(t, y)-g(t, z)| \leqslant D|y-z| \text { for all } t \in J,|y|,|y|,|z| \leqslant R_{0}+r+r^{\prime}, \\
|z-k g(t, z)| \leqslant \rho R_{0} \text { for } t \in J,|z| \leqslant R_{0}, \\
L c_{4}(c+C) \leqslant r, \\
k c_{5}\left(c+D\left(r+r^{\prime}\right)\right) \leqslant\left(1-\rho c_{5}\right) R_{0}, r^{\prime}=0 \text { if } p=q .
\end{gathered}
$$

We have already assumed before that $q \times q$ matrix $M=\left[\left(\omega_{s}, \phi_{i}\right), s, i=1, \cdots, q\right]$ is nonsingular, that $f:[0, a] \rightarrow R$ and $g:[0, a] \times \mathbb{R} \rightarrow \mathbb{R}$ are continuous, and that the coefficients $p_{1}, \cdots, p_{n}$ in (1) are also continuous.

(4.i) For continuous functions $f$ and $g$ satisfying relations (10)-(15), problem (1), (2) has at least one solution $x(t), 0 \leqslant t \leqslant a$, with $\|x\|_{x} \leqslant R_{0}+r+r^{\prime}$. Actually, for $p>q, r^{\prime}>0$, at least one solution for every element $x_{02} \in X_{02},\left\|x_{02}\right\|_{x} \leqslant r^{\prime}$. 
Proof. We understand that if $p=q$ then $r^{\prime}=0$. For $p \geqslant q$ and $r^{\prime}=0$ we take $x_{02}=0, x_{02}$ $\in X_{02}$. If $r^{\prime}>0$ we take any element $x_{02} \in X_{02}$ with $\left\|x_{02}\right\|_{x} \leqslant r^{\prime}$. Thus, $x=x_{01}+x_{02}+x_{1}, x_{01}$ $\in S_{0}, x_{02} \in X_{02}, x_{1} \in X_{1}$. Let us show that $T$ maps $\Omega$ into itself. We note that, for $\left(x_{01}, x_{1}\right) \in$ $\Omega$, hence $\left\|x_{01}\right\|_{\infty} \leqslant R_{0},\left\|x_{1}\right\|_{\infty} \leqslant r$, we certainly have, by (9) and (14),

$$
\left\|\bar{x}_{1}\right\|_{x}=\|H(I-Q)[f(t)+g(t, x(t))]\|_{\infty} \leqslant L c_{4}(c+C) \leqslant r,
$$

and $\bar{x}_{1} \in S_{1}$.

For $x_{01} \in S_{0}$, that is, $\left\|x_{01}\right\|_{x} \leqslant R_{0}$, and $x_{1} \in S_{1}$, we have now, by (9),

$$
\begin{aligned}
\bar{x}_{01}(t) & =\left[x_{01}(t)-S Q x_{01}(t)\right]+S Q\left[x_{01}(t)-k g\left(t, x_{01}(t)\right)\right] \\
& -k S Q f(t)+k S Q\left[g\left(t, x_{01}(t)\right)-g\left(t, x_{01}(t)+x_{02}(t)+x_{1}(t)\right)\right],
\end{aligned}
$$

where $x_{01} \in X_{0}$, hence $S Q x_{01}=x_{01}$, and the first bracket is zero. Moreover $\left|x_{01}(t)\right| \leqslant R_{0}$ and, by (13), $\mid x_{01}(t)-k g\left(t, x_{01}(t) \mid \leqslant \rho R_{0}\right.$. We have now, by (15), $\left|\bar{x}_{01}(t)\right| \leqslant 0+\rho c_{5} R_{0}+k c_{5} c+$ $k c_{5} D\left(r+r^{\prime}\right) \leqslant R_{0}$, and $\bar{x}_{01} \in S$. To complete the proof of (4.i) we must show that $T \Omega$ is compact in the topology of $C[0, a]$. Indeed, for $\left(x_{01}, x_{1}\right) \in \Omega,\left(\bar{x}_{01}, \bar{x}_{1}\right)=T\left(x_{01}, x_{1}\right) \in T \Omega$, then $\tilde{x}_{1}$ is in $X$, and by (3) $\left\|\bar{x}_{1}^{\prime}\right\|_{x} \leqslant \gamma\|f+g\|_{2}=M_{1}$ if $n>1$, and $\left\|\bar{x}_{1}^{\prime}\right\|_{2} \leqslant \gamma\|f+g\|_{2}=M_{1}$ if $n=1$. Thus, for $n>1, x_{1}$ is continuous, bounded by $\left\|\bar{x}_{1}\right\|_{x} \leqslant r$, and uniformly Lipschitzian of fixed constant $M_{1}$. For $n=1$ then $x_{1}$ is again continuous and bounded, and equiabsolutely continuous, as the following usual argument shows. Indeed, for any finite system of nonoverlapping intervals in $[0, a]$, say

$$
\left(\alpha_{i}, \beta_{i}\right), i=1, \ldots, N \text {, with } \sum_{i=1}^{N}\left(\beta_{i}-\alpha_{i}\right) \leqslant \delta \text {, take } F=\bigcup_{i=1}^{N}\left(\alpha_{i}, \beta_{i}\right), \text { meas } F \leqslant \delta,
$$

and then

$$
\sum_{i=1}^{N}\left|\bar{x}_{1}\left(\beta_{i}\right)-\bar{x}_{1}\left(\alpha_{i}\right)\right| \leqslant \int_{F}\left|\bar{x}_{1}^{\prime}(t)\right| \mathrm{d} t \leqslant(\operatorname{meas} F)^{1 / 2}\left(\int_{F}\left|\bar{x}_{1}^{\prime}(t)\right|^{2} \mathrm{~d} t\right)^{1 / 2} \leqslant \delta^{1 / 2} M_{1} .
$$

The elements $\bar{x}_{01}$ of $T \Omega$ are elements of $X_{01}$ which is finite dimensional and they are equibounded since $\left|\bar{x}_{01}(t)\right| \leqslant R_{0}$. Thus, $T \Omega$ is a relatively compact subsct of the closed convex set $\Omega$ in the topology of $X=C[0, a]$. By Schauder's fixed point theorem $T$ has at least one fixed point in $\Omega$. Theorem (4.i) is thereby proved.

\section{AN EXAMPLE}

Let us consider the problem

$$
\begin{aligned}
& E x=x^{\prime \prime \prime}=f(t)+g(t, x(t)), \quad t \in J=[0, a], \\
& x^{\prime \prime}(0)=0, \quad x^{\prime \prime}(a)=0, \quad 2 x(0)+x(a)=0 .
\end{aligned}
$$

The homogeneous problem $x^{\prime \prime \prime}=0$ with the same boundary conditions (17) has the nonzero solution $x(t)=t-a / 3,0 \leqslant t \leqslant a$, and $\operatorname{ker} E=\{c(t-a / 3)\}, p=1$. The homogeneous problem is equivalent to the system $x_{1}^{\prime}=x_{2}, x_{2}^{\prime}=x_{3}, x_{3}^{\prime}=0$ with conditions $x_{3}(0)=0, x_{3}(a)=0,2 x_{1}(0)$ 
$+x_{1}(a)=0$, or

$$
\begin{gathered}
\left(\begin{array}{l}
x_{1}^{\prime} \\
x_{2}^{\prime} \\
x_{3}^{\prime}
\end{array}\right)=\left(\begin{array}{lll}
0 & 1 & 0 \\
0 & 0 & 1 \\
0 & 0 & 0
\end{array}\right)\left(\begin{array}{l}
x_{1} \\
x_{2} \\
x_{3}
\end{array}\right), \\
\left(\begin{array}{lll}
0 & 0 & 1 \\
0 & 0 & 0 \\
2 & 0 & 0
\end{array}\right)\left(\begin{array}{l}
x_{1}(0) \\
x_{2}(0) \\
x_{3}(0)
\end{array}\right)+\left(\begin{array}{lll}
0 & 0 & 0 \\
0 & 0 & 1 \\
1 & 0 & 0
\end{array}\right)\left(\begin{array}{l}
x_{1}(a) \\
x_{2}(a) \\
x_{3}(a)
\end{array}\right)=0 .
\end{gathered}
$$

The dual problem is, therefore,

$$
\begin{gathered}
\left(y_{1}^{\prime} y_{2}^{\prime} y_{3}^{\prime}\right)=-\left(\begin{array}{lll}
y_{1} & y_{2} & y_{3}
\end{array}\right)\left(\begin{array}{ccc}
0 & 1 & 0 \\
0 & 0 & 1 \\
0 & 0 & 0
\end{array}\right), \\
\left(y_{1}(0) y_{2}(0) y_{3}(0)\right)=\left(\begin{array}{llll}
\alpha_{1} & \alpha_{2} & \alpha_{3}
\end{array}\right)\left(\begin{array}{ccc}
0 & 0 & 1 \\
0 & 0 & 0 \\
2 & 0 & 0
\end{array}\right), \\
\left(y_{1}(a) y_{2}(a) y_{3}(a)\right)=-\left(\begin{array}{llll}
\alpha_{1} & \alpha_{2} & \alpha_{3}
\end{array}\right)\left(\begin{array}{ccc}
0 & 0 & 0 \\
0 & 0 & 1 \\
1 & 0 & 0
\end{array}\right)
\end{gathered}
$$

with $\alpha_{1}, \alpha_{2}, \alpha_{3}$ arbitrary, or $y_{1}^{\prime}=0, y_{2}^{\prime}=-y_{1}, y_{3}^{\prime}=-y_{2}$ with conditions $y_{1}(0)+2 y_{1}(a)=0$. $y_{2}(0)=0, y_{2}(a)=0$. Then, $y_{3}^{\prime \prime}=-y_{2}^{\prime}=y_{1}, y_{3}^{\prime \prime \prime}=y_{1}^{\prime}=0$, and by writing $y$ for $y_{3}$, we have the dual problem of $(16,17)$ :

$$
E^{*} y=-y^{\prime \prime \prime}=0 . \quad t \in J=[0, a], \quad y^{\prime}(0)=0, \quad y^{\prime}(a)=0, \quad y^{\prime \prime}(0)+2 y^{\prime \prime}(a)=0 .
$$

This problem has the nonzero solution $y(t)=1,0 \leqslant t \leqslant a$, and ker $E^{*}=\{d\}, q=1$.

By normalization we have

$$
\phi(t)=3 a^{-3 / 2}(t-a / 3), \quad \omega(t)=a^{-1 / 2}, \quad 0 \leqslant t \leqslant a, \quad \operatorname{ker} E=\{c \phi\}, \quad \operatorname{ker} E^{*}=\{d \omega\}, \quad p=q=1 .
$$

Here $\phi$ and $\omega$ do not share regions of positivity and negativity.

The relation $x=H y$ is now defined by the set of equations

$$
\begin{gathered}
x^{\prime \prime \prime}=y, \quad x^{\prime \prime}(0)=0, \quad x^{\prime \prime}(a)=0, \quad 2 x(0)+x(a)=0, \\
\int_{0}^{a} x(t)(t-a / 3) \mathrm{d} t=0, \quad \int_{0}^{a} y(t) \mathrm{d} t=0 .
\end{gathered}
$$

We could express $H$ by an integral operator and estimate $H$ by estimating the kernel. We prefer here to solve problem (18) directly, obtaining the estimates step by step.

First, $x^{\prime \prime}(t)=\int_{0}^{t} y(\alpha) \mathrm{d} \alpha$ because of $x^{\prime \prime}(0)=0$, and then $x^{\prime \prime}(a)=\int_{0}^{a} y(\alpha) \mathrm{d} \alpha=0$ because of last relation (18). Moreover, for $0 \leqslant t \leqslant a / 2,\left|x^{\prime \prime}(t)\right| \leqslant\|y\|_{\infty} a / 2$, and by symmetry the same relation holds in $[a / 2, a]$. Thus, $\left\|x^{\prime \prime}\right\|_{x} \leqslant\|y\|_{\infty} a / 2$. We have now 


$$
x(t)=A+B(t-a / 3)+\int_{a / 3}^{t}(t-\beta) x^{\prime \prime}(\beta) \mathrm{d} \beta, \quad A, B \text { constants, }
$$

and the condition $2 x(0)+x(a)=0$ yields

$$
\begin{gathered}
0=2 x(0)+x(a)=3 A+2 \int_{a / 3}^{0}(-\beta) x^{\prime \prime}(\beta) \mathrm{d} \beta+\int_{a / 3}^{a}(a-\beta) x^{\prime \prime}(\beta) \mathrm{d} \beta, \\
3|A| \leqslant\left\|x^{\prime \prime}\right\|_{x}\left(2 \int_{0}^{a / 3} \beta \mathrm{d} \beta+\int_{a / 3}^{a}(a-\beta) \mathrm{d} \beta\right)=\left\|x^{\prime \prime}\right\|_{x} a^{2} / 3, \\
|A| \leqslant(1 / 9) a^{2}\left\|x^{\prime \prime}\right\|_{x} .
\end{gathered}
$$

From the relation $\int_{0}^{a} x(t)(t-a / 3) \mathrm{d} t=0$ we derive now

$$
\begin{gathered}
A \int_{0}^{a}(t-a / 3) \mathrm{d} t+B \int_{0}^{a}(t-a / 3)^{2} \mathrm{~d} t+\int_{0}^{a}(t-a / 3) \mathrm{d} t \int_{a / 3}^{t}(t-\beta) x^{\prime \prime}(\beta) \mathrm{d} \beta=0 \\
(1 / 9) a^{3}|B| \leqslant(1 / 6) a^{2}|A|+\left(17 a^{4} /(8.81)\right)\left\|x^{\prime \prime}\right\|_{\infty} \\
|B| \leqslant(29 /(8.9)) a\left\|x^{\prime \prime}\right\|_{\infty}
\end{gathered}
$$

By using estimates (20) and (21), and by separating the cases $0 \leqslant t \leqslant a / 3$ and $a / 3 \leqslant t \leqslant a$, we obtain from (19),

$$
|x(t)| \leqslant(65 / 108) a^{2}\left\|x^{\prime \prime}\right\|_{x} \leqslant(65 / 216) a^{3}\|y\|_{x} .
$$

Thus, in the topology of $C$ we have

$$
\|H\| \leqslant(65 / 216) a^{3} \text {. }
$$

To estimate $\|Q\|$ we note that, for $y \in Y$, we have

$$
|(Q y)(t)|=\left|\left(\int_{0}^{a} \omega(\alpha) y(\alpha) \mathrm{d} \alpha\right) \omega(t)\right|=\left(\int_{0}^{a} a^{-1 / 2} y(\alpha) \mathrm{d} \alpha\right) a^{-1 / 2} \leqslant a^{-1}\|y\|_{\infty} \int_{0}^{a} \mathrm{~d} \alpha=\|y\|_{x} .
$$

Thus, $\|Q\| \leqslant 1$ and, since $Q$ is a projection, also $\|Q\|=1$.

To estimate $S$ we note that, for $y \in Y_{0}$, we have $y=d^{\#} \omega=d^{*} a^{-1 / 2}$, hence

$$
\|y\|_{x}=\left|d^{\#}\right| a^{-1 / 2}, \quad\left|d^{\#}\right|=a^{1 / 2}\|y\|_{x} .
$$

On the other hand, we have $q=1$ and

$$
M=\int_{0}^{a} \phi \omega \mathrm{d} t=\int_{0}^{a} a^{-1 / 2} \cdot 3 a^{-3 / 2}(t-a / 3) \mathrm{d} t=1 / 2,
$$

and by the definition of $S$, for $x=S y$, we have $x=d \phi$ with $d=d^{\#} / M$, or

$$
\|x\|_{x}=\|S y\|_{x}=\|d \phi\|_{x}=2 \cdot a^{1 / 2}\|y\|_{x} \cdot\left\|3 a^{-3 / 2}(t-a / 3)\right\|_{x}=4\|y\|_{x},
$$

and thus $\|S\| \leqslant 4$. We conclude with the list of the constants of interest:

$$
\|Q\|=c_{3}=1, \quad\|I-Q\| \leqslant c_{4}=2, \quad\|H\| \leqslant L=(65 / 216) a^{3}, \quad\|S Q\| \leqslant c_{5}=4 .
$$


As a numerical example, let $g: \mathbb{R} \rightarrow \mathbb{R}$ be any function satisfying

$$
\begin{aligned}
& |g(z)| \leqslant 1, \quad\left|g(z)-g\left(z^{\prime}\right)\right| \leqslant(4 / 3)\left|z-z^{\prime}\right| \text { for }|z|,\left|z^{\prime}\right| \leqslant R_{0}+r, \\
& |z-g(z)| \leqslant \rho R_{0} \text { for }|z| \leqslant R_{0} .
\end{aligned}
$$

Thus $C=1, D=4 / 3$. We take $a=0.1, k=1, c=0.1$, so that $L=(65 / 216) a^{3}=0.00030092$, and for $r=0.00067$ we also have

$$
\begin{gathered}
L c_{4}(c+C)=(0.00030092)(2)(0.1+1)=0.000662024<0.00067=r, \\
k c_{5}(c+D r)=4(0.1+(4 / 3)(0.00067))=0.4035733 .
\end{gathered}
$$

For $R_{0}=1.19, \rho=0.165$, we have

$$
\left(1-\rho c_{5}\right) R_{0}=(1-4(0.165))(1.19)=0.4046>0.4035733 .
$$

On the other hand, we require that $|z-g(z)| \leqslant \rho R_{0}$ for $|z| \leqslant R_{0}=1.19$. We see that for $1 \leqslant z \leqslant 1.19$ we have $g(z) \leqslant 1$, and the requirement $|z-g(z)| \leqslant \rho R_{0}$ can be satisfied since $0.19=1.19-1<0.19635=\rho R_{0}$.

Thus, problem (16), (17) has at least one solution $x(t)$ with $|x(t)| \leqslant R_{0}+r=1.19067$ for $a=0.1$, $\|f\|_{x} \leqslant 0.1$, and $g$ satisfying (22) with $R_{0}=1.19, r=0.00067, \rho=0.165$. in other words for any $g$ of Lipschitz constant $4 / 3$, whose graph is within the heavy set box in Fig. 1 .

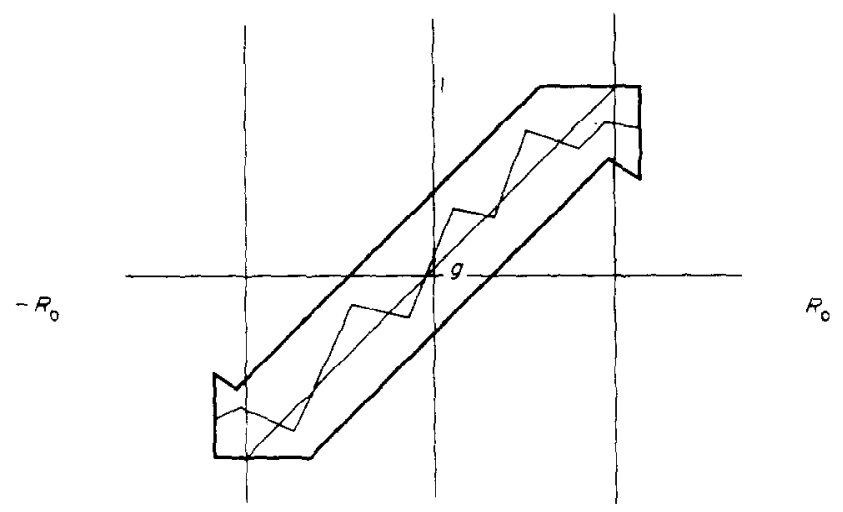

Fig. 1.

\section{ANOTHER EXAMPLE}

Let us consider the problem

$$
\begin{gathered}
E x=x^{\prime \prime \prime}+\lambda^{2} x^{\prime}=f(t)+g(t, x(t)), \quad t \in J=[0, a], \quad \lambda=2 \pi / a, \\
x(a)+\varepsilon x^{\prime}(a)=0, \quad x^{\prime \prime}(a)=0, \quad x^{\prime}(0)-x^{\prime}(a)=0 .
\end{gathered}
$$

The homogeneous problem $x^{\prime \prime \prime}+\lambda^{2} x^{\prime}=0$ with the same boundary conditions (24) has the nonzero solution $x(t)=-\varepsilon \lambda+\sin \lambda t, 0 \leqslant t \leqslant a$, and $\operatorname{ker} E=\{c(-\varepsilon \lambda+\sin \lambda t)\}, p=1$. 
The homogeneous problem is equivalent to the system $x_{1}^{\prime}=x_{2}, x_{2}^{\prime}=x_{3}, x_{3}^{\prime}=-\lambda^{2} x_{2}$ with conditions $x_{1}(a)+\varepsilon x_{2}(a)=0, x_{3}(a)=0, x_{2}(0)-x_{2}(a)=0$, or

$$
\begin{gathered}
\left(\begin{array}{l}
x_{1}^{\prime} \\
x_{2}^{\prime} \\
x_{3}^{\prime}
\end{array}\right)=\left(\begin{array}{ccc}
0 & 1 & 0 \\
0 & 0 & 1 \\
0-\lambda^{2} & 0
\end{array}\right)\left(\begin{array}{l}
x_{1} \\
x_{2} \\
x_{3}
\end{array}\right) \\
\left(\begin{array}{lll}
0 & 0 & 0 \\
0 & 0 & 0 \\
0 & 1 & 0
\end{array}\right)\left(\begin{array}{l}
x_{1}(0) \\
x_{2}(0) \\
x_{3}(0)
\end{array}\right)+\left(\begin{array}{ccc}
1 & \varepsilon & 0 \\
0 & 0 & 1 \\
0 & -1 & 0
\end{array}\right)\left(\begin{array}{l}
x_{1}(a) \\
x_{2}(a) \\
x_{3}(a)
\end{array}\right)=0 .
\end{gathered}
$$

The dual problem is, therefore,

$$
\begin{gathered}
\left(\begin{array}{lll}
y_{1}^{\prime} & y_{2}^{\prime} & y_{3}^{\prime}
\end{array}\right)=-\left(\begin{array}{lll}
y_{1} & y_{2} & y_{3}
\end{array}\right)\left(\begin{array}{ccc}
0 & 1 & 0 \\
0 & 0 & 1 \\
0-\lambda^{2} & 0
\end{array}\right), \\
\left(y_{1}(0) y_{2}(0) y_{3}(0)\right)=\left(\begin{array}{llll}
\alpha_{1} & \alpha_{2} & \alpha_{3}
\end{array}\right)\left(\begin{array}{lll}
0 & 0 & 0 \\
0 & 0 & 0 \\
0 & 1 & 0
\end{array}\right), \\
\left(y_{1}(a) y_{2}(a) y_{3}(a)\right)=-\left(\begin{array}{lll}
\alpha_{1} & \alpha_{2} & \alpha_{3}
\end{array}\right)\left(\begin{array}{ccc}
1 & \varepsilon & 0 \\
0 & 0 & 1 \\
0 & -1 & 0
\end{array}\right),
\end{gathered}
$$

with $\alpha_{1}, \alpha_{2}, \alpha_{3}$ arbitrary, or $y_{1}^{\prime}=0, y_{2}^{\prime}=-y_{1}+\lambda^{2} y_{3}, y_{3}^{\prime}=-y_{2}$ with conditions $y_{3}(0)=0$, $y_{1}(0)=0, y_{2}(a)=\varepsilon y_{1}(a)+y_{2}(0)$. Then, $y_{3}^{\prime \prime}=-y_{2}^{\prime}=y_{1}-\lambda^{2} y_{3}, y_{3}^{\prime \prime \prime}=y_{1}^{\prime}-\lambda^{2} y_{3}^{\prime}=-\lambda^{2} y_{3}^{\prime}$, and by writing $y$ for $y_{3}$, we have the dual problem of (23), (24):

$$
\begin{gathered}
E^{*} y=-y^{\prime \prime \prime}-\lambda^{2} y^{\prime}=0, \quad t \in J=[0, a], \\
y(0)=0, \quad y^{\prime \prime}(0)=0, \quad y^{\prime}(0)-y^{\prime}(a)=\varepsilon\left(y^{\prime \prime}(a)+\lambda^{2} y(a)\right) .
\end{gathered}
$$

This problem has the nonzero solution $y(t)=\sin \lambda t, 0 \leqslant t \leqslant a$, and $\operatorname{ker} E^{*}=\{d \sin \lambda t\}$, $q=1$. By normalization we have

$$
\begin{gathered}
\phi(t)=2^{1 / 2} a^{-1 / 2}\left(1+2 \lambda^{2} \varepsilon^{2}\right)^{-1 / 2}(-\varepsilon \lambda+\sin \lambda t), \quad \omega(t)=2^{1 / 2} a^{-1 / 2} \sin \lambda t, \quad 0 \leqslant t \leqslant a, \\
\operatorname{ker} E=\{c \phi(t)\}, \quad \operatorname{ker} E^{*}=\{d \omega(t)\}, \quad p=q=1 .
\end{gathered}
$$

Here $\phi$ and $\omega$ do not share regions of positivity and negativity.

The relation $x=H y$ is now defined by the set of equations

$$
\begin{gathered}
x^{\prime \prime \prime}+\lambda^{2} x^{\prime}=y, \quad x(a)+\varepsilon x^{\prime}(a)=0, \quad x^{\prime \prime}(a)=0, \quad x^{\prime}(0)-x^{\prime}(a)=0, \\
\int_{0}^{a} x(t)(-\varepsilon \lambda+\sin \lambda t) \mathrm{d} t=0, \quad \int_{0}^{a} y(t) \sin \lambda t \mathrm{~d} t=0 .
\end{gathered}
$$

The differential equation yields

$$
x(t)=A+B \cos \lambda t+C \sin \lambda t+c_{1}(t)+c_{2}(t) \cos \lambda t+c_{3} \sin \lambda t,
$$




$$
\begin{gathered}
c_{1}(t)=\int_{a}^{t} \lambda^{-2} y(\alpha) \mathrm{d} \alpha, \quad c_{2}(t)=-\int_{a}^{t} \lambda^{-2} y(\alpha) \cos \lambda \alpha \mathrm{d} \alpha, \\
c_{3}(t)=-\int_{a}^{t} \lambda^{-2} y(\alpha) \sin \lambda \alpha \mathrm{d} \alpha .
\end{gathered}
$$

The condition $x^{\prime \prime}(a)=0$ implies $B=0$. Since $\lambda=2 \pi / a, \sin \lambda t$ and $\cos \lambda t$ are periodic of period $a$, we have

$$
x^{\prime}(0)-x^{\prime}(a)=\lambda c_{3}(0)=\lambda^{-1} \int_{a}^{0} y(\alpha) \sin \lambda \alpha \mathrm{d} \alpha=0,
$$

and the condition $x^{\prime}(0)-x^{\prime}(a)=0$ is satisfied. The condition $x(a)+\varepsilon x^{\prime}(a)=0$ yields $A+$ $\varepsilon \lambda C=0$; hence $A=-\varepsilon \lambda C$, and

$$
x(t)=(-\varepsilon \lambda+\sin \lambda t) C+c_{1}(t)+c_{2}(t) \cos \lambda t+c_{3}(t) \sin \lambda t
$$

Now the condition $\int_{0}^{a} x(t)(-\varepsilon \lambda+\sin \lambda t) \mathrm{d} t=0$ yields

$$
0=C a\left(2^{-1}+\lambda^{2} \varepsilon^{2}\right)+\int_{0}^{a}[-\varepsilon \lambda+\sin \lambda t]\left[c_{1}(t)+c_{2}(t) \cos \lambda t+c_{3}(t) \sin \lambda t\right] \mathrm{d} t
$$

from which we can derive $C$. An estimate for $C$ is then

$$
\begin{aligned}
|C| & \leqslant 2 a^{-1}\left(1+2 \lambda^{2} \varepsilon^{2}\right)^{-1}(1+\varepsilon \lambda) \lambda^{-2} \int_{0}^{a} \mathrm{~d} t\left|\int_{a}^{t} y(\alpha) \mathrm{d} \alpha+\int_{a}^{t} y(\alpha) \cos \lambda(t-\alpha) \mathrm{d} \alpha\right| \\
& \leqslant 2 a^{-1}\left(1+2 \lambda^{2} \varepsilon^{2}\right)^{-1}(1+\varepsilon \lambda) \lambda^{-2}\|y\|_{\infty} \int_{0}^{a} \mathrm{~d} t \int_{t}^{a} 2 \mathrm{~d} \alpha \\
& =2 a \lambda^{-2}\left(1+2 \varepsilon^{2} \lambda^{2}\right)^{-1}(1+\varepsilon \lambda)\|y\|_{x}
\end{aligned}
$$

Finally,

Thus,

$$
\begin{aligned}
|x(t)| & =\left|(-\varepsilon \lambda+\sin \lambda t) C+c_{1}(t)+c_{2}(t) \cos \lambda t+c_{3}(t) \sin \lambda t\right| \\
& \leqslant(1+\varepsilon \lambda)|C|+\lambda^{-2}\left|\int_{a}^{t} y(\alpha) \mathrm{d} \alpha+\int_{a}^{t} y(\alpha) \cos \lambda(t-\alpha) \mathrm{d} \alpha\right| \\
& \leqslant(1+\varepsilon \lambda) \cdot 2 a \lambda^{-2}\left(1+2 \varepsilon^{2} \lambda^{2}\right)^{-1}(1+\varepsilon \lambda) \mid\left(y\left\|_{x}+\lambda^{-2} 2 a\right\| y \|_{x}\right.
\end{aligned}
$$

$$
\|H\| \leqslant \lambda^{-2} a\left(2\left(1+2 \lambda^{2} \varepsilon^{2}\right)^{-1}(1+\varepsilon \lambda)^{2}+2\right) .
$$

Since $\lambda=2 \pi / a$ we also have

$$
\|H\| \leqslant(2 \pi)^{-2} a^{3}\left(2+2^{-1}\left(1+2 \lambda^{2} \varepsilon^{2}\right)^{-1}(1+\varepsilon \lambda)^{2}\right) .
$$

To estimate $\|Q\|$ we note that, for any $y \in Y$, we have

$$
\begin{aligned}
|Q y(t)| & =\left|\left(\int_{0}^{a} \omega(\alpha) y(\alpha) \mathrm{d} \alpha\right) \omega(t)\right| \\
& =\left|\left(\int_{0}^{a} 2^{1 / 2} a^{-1 / 2} y(\alpha) \sin \lambda \alpha \mathrm{d} \alpha\right) 2^{1 / 2} a^{-1 / 2} \sin \lambda t\right| \\
& \leqslant 2 a^{-1}\left|y\left\|_{\infty} \int_{0}^{a}|\sin \lambda \alpha| \mathrm{d} \alpha=2 a^{-1}\right\| y\left\|_{\infty} \cdot 4 / \lambda=(4 / \pi)\right\| y \|_{\infty},\right.
\end{aligned}
$$


and hence $\|Q\| \leqslant 4 / \pi$. To estimate $\|S\|$ we note that, for $y \in Y_{0}$, we have $y=d^{*} \omega=$ $d^{\#} 2^{1 / 2} a^{-1 / 2} \sin \lambda t$, hence

$$
\|y\|_{\infty}=\left|d^{\#}\right| 2^{1 / 2} a^{-1 / 2}, \quad\left|d^{\#}\right|=2^{-1 / 2} a^{1 / 2}\|y\|_{x} .
$$

On the other hand, we have $q=1$ and

$$
\begin{aligned}
M=\int_{0}^{a} \phi \omega \mathrm{d} t & =\int_{0}^{a} 2^{1 / 2} a^{-1 / 2}\left(1+2 \lambda^{2} \varepsilon^{2}\right)^{-1 / 2}(-\varepsilon \lambda+\sin \lambda t) \cdot 2^{1 / 2} a^{-1 / 2} \sin \lambda t \mathrm{~d} t \\
& =\left(1+2 \lambda^{2} \varepsilon^{2}\right)^{-1 / 2},
\end{aligned}
$$

and by the definition of $S$, for $x=S y$ we have $x=d \phi$ with $d=d^{\#} / M$, or

$$
\begin{gathered}
\|x\|_{x}=\|S y\|_{x}=\|d \phi\|_{x}=\| 2^{1 / 2} a^{-1 / 2}\left(1+2 \lambda^{2} \varepsilon^{2}\right)^{-1 / 2}(-\varepsilon \lambda+\sin \lambda t) \cdot 2^{-1 / 2} a^{1 / 2} \\
\times\|y\|_{x}\left(1+2 \lambda^{2} \varepsilon^{2}\right)^{1 / 2}\left\|_{x}=\right\| \varepsilon \lambda+\sin \lambda t\left\|_{x}=(1+\lambda \varepsilon)\right\| y \|_{x},
\end{gathered}
$$

and thus $\|S\| \leqslant 1+\lambda \varepsilon$. We conclude with the list of the constants of interest:

$$
\begin{aligned}
& \|Q\| \leqslant c_{3}=4 / \pi, \quad\|I-Q\| \leqslant 1+4 / \pi, \quad\|S Q\| \leqslant c_{5}=(1+\lambda \varepsilon)(4 / \pi), \\
& \|H\| \leqslant L=(2 \pi)^{-2} a^{3}\left(2+2^{-1}\left(1+2 \lambda^{2} \varepsilon^{2}\right)^{-1}(1+\varepsilon \lambda)\right)^{2} .
\end{aligned}
$$

As a numerical example, let $g: \mathbb{R} \rightarrow \mathbb{R}$ be any function satisfying

$$
\begin{gathered}
|g(z)|<1, \quad\left|g(z)-g\left(z^{\prime}\right)\right| \leqslant(4 / 3)\left|z-z^{\prime}\right| \text { for }|z|,\left|z^{\prime}\right| \leqslant R_{0}+r, \\
|z-g(z)| \leqslant \rho R_{0} \text { for }|z| \leqslant R_{0} .
\end{gathered}
$$

Thus, $C=1, D=4 / 3$. We take $a=0.4, k=1, \lambda=2 \pi / a=12.566, \varepsilon=0.01 . \lambda \varepsilon=0.12566$. $c_{4}=1+4 / \pi=2.27336, c_{5}=(1+\lambda \varepsilon)(4 / \pi)=1.4332, L=0.0068148, c=0.1 . r=0.018$ we have

$$
\begin{aligned}
& L c_{4}(c+C)=(0.0068148)(2.27324)(0.1+1)=0.017049<0.018=r, \\
& k c_{5}(c+D r)=(1.4332)(0.1+(4 / 3)(0.018))=0.17772 .
\end{aligned}
$$

For $R_{0}=1.5, \rho=0.5$ we have

$$
\left(1-\rho c_{5}\right) R_{0}=(1-(0.5)(1.4332))(1.5)=0.4251>0.17772 \text {. }
$$

while $R_{0}-1=0.5<0.75=(0.5)(1.5)=\rho R_{0}$.

Thus, problem (23), (24) has at least a solution $x(t)$ with $|x(t)| \leqslant R_{0}+r=1.518$ for $a=$ $0.5,\|f\|_{x} \leqslant 0.1$ and any $g$ satisfying (25) with $R_{0}=1.5, r=0.018, \rho=0.5$, in other words, for $g$ of Lipschitz constant $4 / 3$ whose graph is within the heavy box in Fig. 2.

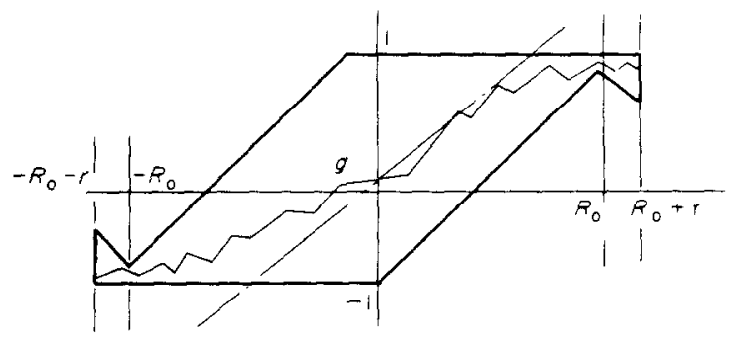

Fig. 2. 


\section{APPENDIX}

The operator bounds used in this paper are obtained by functional analysis considerations. An elementary analysis of the same operators is contained in previous papers of the authors $[1,2,6]$, and we think it relevant for the reader to see briefly the latter in the form which is needed here.

Let us consider the real linear system

$$
\begin{aligned}
& u^{\prime}-A(t) u=h(t), \quad 0 \leqslant t \leqslant a, \\
& B_{1} u(0)+B_{2} u(a)=0,
\end{aligned}
$$

where $u(t)=\operatorname{col}\left(u_{1}, \ldots, u_{n}\right), h(t)=\operatorname{col}\left(h_{1}, \ldots, h_{n}\right), A=\left[c_{i j}(t)\right]$ is an $n \times n$ matrix with bounded measurable entries, and $B_{1}, B_{2}$ are $m \times n$ matrices, $0 \leqslant m \leqslant n$. The corresponding homogeneous system is then

$$
u^{\prime}-A(t) u=0, B_{1} u(0)+B_{2} u(a)=0 .
$$

The adjoint system, in parametric form is therefore (see, e.g. [6, p. 32] also for references).

$$
\mathrm{d} \tilde{v} / \mathrm{d} t+\dot{v} A(t)=0, \tilde{v}(0)=\bar{\alpha} B_{1}, \quad \bar{v}(a)=-\dot{\alpha} B_{2},
$$

where $v(t)=\operatorname{col}\left(v_{1}, \ldots, v_{n}\right)$, where $\alpha=\operatorname{col}\left(\alpha_{1}, \ldots, \alpha_{n}\right)$ is an arbitrary constant matrix, and where $\dot{M}$ denotes the transpose of any matrix $M$.

If $E$ denotes the differential operator with boundary conditions defined by relations (27), then ker $E$ is the set of all solutions of problem (28). Analogous definitions have $E^{*}$ and $\operatorname{ker} E^{*}$ for relations (29).

For any measurable $n$-vector $z(t)=\left(z_{1}, \ldots, z_{n}\right), 0 \leqslant t \leqslant a$, we denote by $\|z\|_{x}$ the usual norm $\|z\|_{x}=$ Ess Sup $_{0 \leqslant i \leqslant a}|z(t)|$, where $|z|$ denotes the Euclidean norm of $z$. If $z \in\left(L_{\nu}[0, a]\right)^{n}, \nu \geqslant 1$, let $\|z\|_{\nu}$ denote the $L_{v}$-norm $\|z\|_{\nu}=$ $a^{-1}\left(\int_{0}^{a}|z(t)|^{\nu} \mathrm{d} t\right)^{1 / \nu}$

Let $Y$ denote the $L_{\nu}$-space of $n$-vectors $v(t)=\left(v_{1}, \ldots, v_{n}\right)$ with $|v(t)| L_{v}$-integrable, and let $Y$ be equipped with the norm \|\|$_{1}$.

Let $X$ denote the (Sobolev) space of all $n$-vectors $u(t)=\left(u_{1}, \ldots, u_{n}\right), 0 \leqslant t \leqslant a$, whose elements are $A C$ in $[0, a]$, and whose derivatives $u^{\prime}(t)=\left(u_{1}^{\prime}, \ldots, u_{n}^{\prime}\right)$ are $L_{v}$-integrable in $[0, a]$, and let $X$ be equipped with the norm $\|u\|_{\nu}^{1}=$ $\|u\|_{x}+\left\|u^{\prime}\right\|_{z}$.

Let $U$ be the $n \times p$ matrix, whose $p$ columns form a basis for the solutions to the given boundary value problem (28), $0 \leqslant p \leqslant n$, and let $V$ be a $q \times n$ matrix whose $q$ rows form a basis for the solutions of the adjoint boundary value problem (29), $0 \leqslant q \leqslant n$. Let $c$ and $d$ denote the $p \times p$ and $q \times q$ matrices whose entries are numbers defined by

$$
c=\int_{0}^{a} \dot{U}(s) U(s) \mathrm{d} s, \quad d=\int_{0}^{a} V(s) \bar{V}(s) \mathrm{d} s .
$$

These matrices are nonsingular (cf. [6, p. 35]). Let $P: X \rightarrow X$ and $Q: Y \rightarrow Y$ be the projection operators defined

$$
\begin{array}{lll}
(P u)(t)=U(t) \alpha, & \alpha=c^{-1} \int_{0}^{a} \tilde{U}(s) u(s) \mathrm{d} s, & u \in X, \\
(Q v)(t)=\bar{V}(t) \beta, & \beta=d^{-1} \int_{0}^{a} V(s) v(s) \mathrm{d} s, & v \in Y,
\end{array}
$$

(for $p=0$ take $P=0$, for $q=0$ take $Q=0$ ). The operators $P, Q$ are projection operators in the Banach spaces $X$ and $Y$ respectively in the sense that they are linear are

for suitable constants $\lambda_{1}, \lambda_{2}$.

$$
\begin{array}{lll}
P P=P, & \|u\|_{\nu}^{1} \leqslant\|P u\|_{\nu} \leqslant \lambda_{1}\|u\|_{\nu}, & u \in X, \\
Q Q=Q, & \|v\|_{\nu} \leqslant\|Q v\|_{\nu} \leqslant \lambda_{2}\|v\|_{\nu}, & v \in Y,
\end{array}
$$

Let $X_{0}=P X, X_{1}=(I-P) X, Y_{0}=Q Y, Y_{1}=(I-Q) Y$, so that $X$ and $Y$ have the decompositions $X=X_{0}+X_{1}$, $Y=Y_{0}+Y_{1}$, and thus, for instance, $P X=0$ if and only if $x \in X_{1}, Q v=0$ if and only if $v \in Y_{1}$. Moreover, we have here $X_{0}=\operatorname{ker} E, Y_{0}=\operatorname{ker} E^{*}$.

The following theorem holds.

THEOREM A.1. If $h \in Y=\left(L_{\nu}[0, a]\right)^{n}, \nu \geqslant 1$, then the boundary value problem (26), (27) has solutions $u \in X$ if and only if $Q h=0$. If $Q h=0$, then the boundary value problem (26), (27) has a unique solution $K h$ with $P K h=0$, and all other solutions are of the form $K h+U a, a$ an arbitrary row $p$-vector. Furthermore the linear map $K: Y_{1} \rightarrow X_{1}$ is a bounded linear map in the norms of $X_{1}$ and $Y_{1}$.

This, which is derived from Fredholm alternative, was proved, for example, in [6] for $\nu=1$. The proof is analogous for any $\nu \geqslant 1$. We refer to [6, pp, 32-39]. Thus, for any $h \in Y=\left(L_{\nu}[0, a]\right)^{n}$, with $Q h=0$ (that is, $\left.h \in Y_{1}\right)$, the only solution $u(t)=K h$ of problem (1) with $P u=0$ satisfies $\|u\|_{\nu}^{1} \leqslant \gamma\left\|_{\nu}\right\|_{\nu}$ for some constant $\gamma$ independent of $h$. 
As we know, the analogous problem

$$
\begin{aligned}
& x^{(n)}+p_{1}(t) x^{(n-1)}+\ldots+p_{n}(t) x=f(t), \quad 0 \leqslant t \leqslant a, \\
& \sum_{i=0}^{n-1}\left(a_{i j} x^{(j)}(0)+b_{i j} x^{(j)}(a)\right)=0, \quad i=1, \ldots, m,
\end{aligned}
$$

where $a_{i}, b_{i j}$ are constants, and $p_{1}(t), \ldots, p_{n}(t)$ bounded and measurable functions in $[0, a]$, that is, problem $E x=$ $f(t)$ with the notations of No. 2 , is reduced to problem $(26)$, (27) by the transformation $u_{1}=x, \ldots, u_{n}=x^{(n-1)}$. namely

$$
\begin{aligned}
& u_{1}^{\prime}-u_{2}=0, \ldots, u_{n-1}^{\prime}-u_{n}=0, u_{n}^{\prime}+p_{n}(t) u_{1}+\ldots+p_{1}(t) u_{n}=f(t), \\
& \sum_{j=1}^{n}\left(a_{i, j-1} u_{j}(0)+b_{i, j-1} u_{j}(a)\right)=0, i=1, \ldots, m .
\end{aligned}
$$

In other words, $h(t)=\operatorname{col}(0,0, \ldots, 0, f(t))$, and if $Q h=0$, then there is one and only one solution $x=K h$ of (32) with $P K h=0$. Any other solution is of the form $u=K h+U c$, where $c$ is any $p$ column vector. Finally, $K: Y_{1} \rightarrow X_{1}$ is a bounded linear map in the norms \|\|$_{\nu}$ of $Y_{1}$ and \|\|$_{\nu}^{1}$ of $X_{1}$, so that

$$
\|K h\|_{\nu}^{1} \leqslant \gamma\|h\|_{\nu}
$$

Thus, for $z=K h$,

$$
\|z\|_{\nu}^{1}=\left\|\left(z_{1}, \ldots, z_{n}\right)\right\|_{x}+\left\|\left(z_{1}^{\prime}, \ldots, z_{n}^{\prime}\right)\right\|_{\nu} \leqslant \gamma\|h\|_{\nu},
$$

and for the particular system (32) we derive

$$
\left\|z_{1}\right\|_{x},\left\|z_{1}^{\prime}\right\|_{x}, \ldots,\left\|z_{1}^{(n)}\right\|_{x} \leqslant \gamma\|f\|_{\nu},\left\|z_{l}^{(n)}\right\|_{\nu} \leqslant \gamma\|f\|_{\nu} .
$$

The adjoint system is

$$
v_{1}^{\prime}-p_{n} v_{n}, v_{2}^{\prime}+v_{1}-p_{n-1} v_{n}, \ldots, v_{n}^{\prime}+v_{n-1}=p_{1} v_{n}
$$

which can be reduced to the differential equation

$$
v_{n}^{(n)}-(-1)^{n-1} p_{n} v_{n}-(-1)^{n-2}\left(p_{n-1} v_{n}\right)^{\prime}-\ldots+\left(p_{2} v_{n}\right)^{(n-2)}-\left(p_{1} v_{n}\right)^{(n-1)}=0 \text {. }
$$

If $y(t)=\operatorname{col}\left(y_{1}, \ldots, y_{n}\right)$ is any element of ker $E^{*}$, then $y_{n}=\omega \in \operatorname{ker} E^{*}$ of Section 2, and the orthogonality of $h(t)$ with $y$, or $\int_{0}^{a}\left(h_{1} y_{1}+\ldots+h_{n} y_{n}\right) \mathrm{d} t=0$, reduces to

$$
\int_{0}^{a} f(t) \omega(t) \mathrm{d} t=0
$$

On the other hand, the orthogonality of $K h=\left(z_{1}, \ldots, z_{n}\right)$ with any element $x(t)=\operatorname{col}\left(x_{1}, \ldots, x_{n}\right)$ of ker $E$. or $\int_{0}^{a}\left(z_{1} x_{1}+\ldots+z_{n} x_{n}\right) \mathrm{d} t=0$, may not be the same as the orthogonality of $z_{1}(t)$ with $x_{1}(t)=\phi(t) \in$ ker $E$ of Section 2, or $\int_{0}^{a} z_{1}(t) \phi(t) \mathrm{d} t=0$. Actually, if $\phi_{1}, \ldots, \phi_{p}$ denotes the base of ker $E$ we have chosen in Section 2, we may well assume $\phi_{1}, \ldots, \phi_{p}$ to be the first row of $U$, and we have only to determine the solution $x(t)=z_{1}(t)+$ $\phi_{1} a_{1}+\ldots+\phi_{p} a_{p}$ which satisfies $\int_{0}^{a} x(t) \phi_{i}(t) \mathrm{d} t=0, i=1, \ldots, p$. We obtain

or

$$
\int_{0}^{a} z_{1}(t) \phi_{i}(t) \mathrm{d} t+a_{i}=0, i=1, \ldots, p,
$$

Hence

$$
x(t)=z_{1}(t)-\left(z_{1}, \phi_{1}\right) \phi_{1}(t)-\ldots-\left(z_{1}, \phi_{p}\right) \phi_{p}(t)=(I-P) z_{1}(t) .
$$

$$
x^{(j)}(t)=z_{1}^{(j)}(t)-\left(z_{1}, \phi_{1}\right) \phi_{1}^{(j)}(t)-\ldots-\left(z_{1}, \phi_{p}\right) \phi_{p}^{(j)}(t), j=0,1, \ldots, n,
$$

Here all functions $p_{1}, \ldots, p_{n}$ are bounded, say $\left|p_{i}(t)\right| \leqslant \mu$, all functions $\phi_{i}^{(j)}(t), j=0,1, \ldots, n-1$, are also bounded, say $\left|\phi^{(j}(t)\right| \leqslant M$, and

Thus,

$$
\left\|z_{1}^{(j)}(t)\right\|_{x} \leqslant \gamma\|f\|_{\nu}, \quad j=0,1, \ldots, n-1 .
$$

$$
\left\|x^{(i)}(t)\right\|_{x} \leqslant \gamma\|f\|_{\nu}+\gamma\|f\|_{\nu} \cdot p a M^{2}=\gamma\|f\|_{\nu}\left(1+p a M^{2}\right), \quad j=0,1, \ldots, n,
$$

This proves statement (3) in Section 2

\section{REFERENCES}

1. Bowman T. T., Bifurcation of solutions of nonlinear boundary value problems, Nonlinear Analysis 5, 655-667 (1981). 
2. Bowman T. T., Volterra series and the alternative method, in Dynamical Systems $I I$ (Edited by A. R. BEDNAREK and L. CesARI), pp. 1-10, Academic Press, New York (1982).

3. Cesari L.. Functional analysis and periodic solutions of nonlinear differential equations, Contrib. diff. Eqns 1, 149-187 (1963).

4. Cesari L., Functional analysis and Galerkin's method, Michigan Math. J. 11. 335-414 (1964).

5. CeSARI L.. Existence in the large of periodic solutions of hyperbolic partial differential equations, Archs ration. Mech. Analysis 20, 170-190 (1965).

6. CESARI L., Functional analysis, nonlinear differential equations. and the alternative method, in Nonlinear Functional Analysis and Differential Equations (Edited by L. CESARI. R. KANNAN and J. D. SCHUUR), pp. 1-196. Dekker, New York (1976).

7. CESARI L., Nonlinear problems across a point of resonance for nonselfadjoint systems in Nonlinear Analysis. A Volume in Honor of E. H. Rothe, pp. 43-67, Academic Press, New York (1978).

8. Cesari L. \& Kannan R., Periodic solutions of nonlinear wave equations, Archs ration. Mech. Analysis 82, 295312 (1983).

9. Cesari L. \& Pucci P., Global periodic solutions of the nonlinear wave equation, Archs ration. Mech. Analysis (to appear).

10. DE Figleiredo D. G. The Dirichlet problem for nonjinear elliptic equations. A Hilbert space approach/Partial differential equations and related topics. Lecture Notes Mathematics 446, 144-165. Springer. Berlin (1975).

11. LANDESMAN E. M. \& LAZER A. C., Nonlinear perturbations of linear elliptic boundary value problems at resonance. J. Math. Mech. 19.609-623 (1970).

12. LOCKER J., An existence analysis for nonlinear equations in Hilbert spaces, Trans. Am. math. Soc. 128, 403-413 (1967).

13. LOCKER J., An existence analysis for nonlinear boundary value problems, SIAM J. appl. Math. 19. 199-207 (1970).

14. SHAW H. C., A nonlinear elliptic boundary value problem at resonance. J. diff. Eqns 26. 335-346 (1977).

15. Williams S. A.. A sharp sufficient condition for solutions of a nonlinear elliptic boundary value problem. $J$. diff. Eqns 8, 580-586 (1970). 